\title{
Detection and antibiotic resistance pattern of Staphylococcus aureus and (MRSA) isolated from healthcare workers Nares at three Gaza hospitals, Palestine
}

Nahed Al Laham

Department of Laboratory Medicine, Faculty of Applied Medical Sciences, Al Azhar university-Gaza, Gaza Strip, Palestine.

Corresponding author:

Dr. Nahed Al Laham

”n.lahamm@alazhar.edu.ps, dr.allaham@hotmail.com

\section{Abstract}

Background: Staphylococcus aureus is a significant cause of nosocomial morbidity and mortality and has been identified as an emerging problem in the community. Here we detect the carriage rate and antibiotic resistance pattern of $S$. aureus and MRSA isolated from 140 healthcare workers (HCWs) nares at Gaza hospitals.

Methods: A cross sectional study was conducted on a total of 140 HCWs. S. aureus were recovered and identified from nasal swabs by conventional and molecular laboratory techniques. Susceptibility to 13 commonly used antibiotics against $S$. aureus infections was determined by disk diffusion test according to the CLSI guidelines. Testing by PCR for the presence of nuc, mecA, cap 5 and cap 8 genes was performed on all isolates.

Results: The nasal carriage rate of $S$. aureus was 42.1\% (59/140), of MRSA was $22.6 \%$ (32/140) and of MSSA was 19.3\% (27/140), with the highest carriage rate of MRSA among HCWs from surgery wards. There was no significant difference between the gender or hospitals with regard to $S$. aureus and MRSA carriage rate. However, a statistically significant difference was found for ward type and work experience. All 59 strains of $S$. aureus were sensitive to vancomycin and doxycycline, while nearly all were resistant to penicillin and ampicillin. The antibiotic resistance among MRSA was much higher than that among MSSA with a statistically significant difference for most antibiotics tested. Nearly $61 \%$ of isolates were noted to be multidrug resistant with higher percentage in MRSA (24/32; 75\%) in comparison to MSSA 
$(12 / 27 ; 44,44 \%)$. All isolates were successfully genotyped for capsular polymorphism type 5 (55.9\%) and type 8 (44.1\%).

Conclusion: This is the first study in Gaza Strip investigating the nasal carriage of S. aureus among HCWs. High rate of MRSA isolates were shown to be MDR. These results highlight the critical need for screening and tracking MRSA among HCWs and to monitor and manage the usage of antibiotics in the hospitals and community in Gaza, Palestine.

Keywords: S. aureus, MRSA, HCWs, Antibiotic resistance pattern, Gaza, Palestine

\section{Introduction}

Staphylococcus aureus is one of the most prevalent human pathogens isolated from hospitalized patients worldwide, and is of increasing importance in the community setting. It causes a wide range of localized and systemic diseases ranging from skin and soft-tissue infections to infective endocarditis, osteomyelitis, bacteremia and necrotizing pneumonia, as well as various toxin and superantigen-mediated diseases [1-4]. Methicillinresistant Staphylococcus aureus (MRSA) is one of the most significant health care associated ( $\mathrm{HA})$ and community-associated (CA) pathogen, causing a wide range of infections and are associated with higher morbidity, mortality, prolonged length of hospital stay and higher healthcare costs as compared to methicillin-susceptible staphylococci $[1,5]$. In recent decades, MRSA has emerged as the most frequently identified antibiotic-resistant pathogen in many parts of the world, including North Africa and the Middle East[6]. Fortunately, whereas HA-MRSA isolates are generally multidrug resistant, CA-MRSA tends to be resistant primarily to $\beta$-lactam antibiotics. However, recent studies indicate that many CA-MRSA lineages are becoming increasingly resistant to non $\beta$-lactam antibiotics [7], while a growing number of reports describe replacement of HA-MRSA by CA-MRSA in healthcare settings $[7,8]$.

S. aureus permanently colonizes the anterior nares of about $20 \%$ to $30 \%$ of the general population. Health care workers (HCWs) are more likely to be colonized than persons in the general population, presumably because of increased exposure, So, HCWs constitute an important reservoir of $S$. aureus and MRSA $[9,10]$. Nasal colonization with S. aureus in HCWs has been associated with an increased risk of invasive $S$. aureus infection $[9,11]$. Increased rates of MRSA colonization among HCWs have at least 3 important implications. First, HCWs may become infected by their own MRSA carriage strains; second, they could serve as a route of cross-transmission to patients; and finally, they may introduce the pathogen into their communities [12]. Several studies in Arabic countries and worldwide have reported that the rate of the nasal carriage of $S$. aureus and MRSA among HCWs ranges from 
$10.1 \%-76 \%$ [10,12-26]. The screening of nasal carriage in HCWs is an important component in the control of MRSA in any health care facility [27].

Epidemiological data about S. aureus and MRSA in HCWs in Palestine are generally scarce and insufficient $[16,28]$ with current knowledge regarding the epidemiology of $S$. aureus in Gaza Strip based on two recent studies; a hospital-acquired and a community-based carriage respectively $[29,30]$.

To that end, we aimed to determine the prevalence of asymptomatic nasal carriage of $S$. aureus and MRSA among HCWs who work in environments with varying degrees of exposure to patients and to determine its susceptibility profile to most common used antibiotics in our hospitals. To the best of our knowledge, this is the first study reporting the prevalence of $S$. aureus and MRSA in HCWs within the Gaza hospitals.

\section{Methods}

\section{Study setting and sampling}

This is a cross-sectional study of a convenience sample consisting of $140 \mathrm{HCWs}$ that was conducted for six months between October 2010 and March 2011. The study was performed at three hospitals in Gaza Strip, two main hospitals (Al-Shifa, the largest public tertiary hospital in Gaza city and EuropeanGaza hospital in Rafah city), as well as from another public primary-care hospital (Odwan hospital in northern Gaza). HCWs including physicians, nurses, and medical technicians who are full-time workers from departments of surgery, internal medicine and intensive care units were involved in this study.

All HCWs included were healthy and had not used antibiotics in past two months prior to sample collection. Participation was on voluntary basis and written informed consent was taken from all participants prior to enrolment. A simple questionnaire was used to obtain demographic data including age, sex, place of work, employment status and length of healthcare service, where data were recorded and analyzed anonymously. Ethical approval from the Helsinki committee at the ministry of health in Gaza Strip (Approval no. PHRC/HC/36/14) was obtained for performing the current study.

Sterile cotton swabs moistened with sterile saline were used for sample collection. The sample was obtained by rotating the swabs gently for five times on both nares so that the tip is entirely at the nasal osteum level [31], and it was directly streaked onto mannitol salt agar and blood agar plates (HiMedia Labs, Mumbai, India). Plates were incubated aerobically at $37^{\circ} \mathrm{C}$ for $24-48 \mathrm{hrs}$.

\section{Isolation and Identification of S. aureus and MRSA}

Conventional techniques: $S$. aureus isolates were identified phenotypically based on colonial morphology, Gram stain, and by using one or more of the following methods: catalase test, tube coagulase test, Pastorex ${ }^{\mathrm{TM}}$ Staph Plus latex agglutination (BioRad, Hercules, California), and the Staph ID 32 API system (bioMérieux, France). Screening for MRSA was done by testing for oxacillin $(1 \mu \mathrm{g})$ resistance using the Kirby-Bauer disc diffusion method as per instructions of Clinical and Laboratory Standards Institute (CLSI) (2009) [32]. Briefly, a 0.5 McFarland suspension of each $S$. aureus strain was streaked onto Mueller Hinton agar plate (HiMedia Labs, Mumbai, India) supplemented with $2 \%$ sodium chloride and incubated for $24 \mathrm{~h}$ at $35^{\circ} \mathrm{C}$. The zone of inhibition was measured and isolates that have zones $<10 \mathrm{~mm}$ diameter were considered to be oxacillin resistant and presumptively diagnosed as MRSA.

Molecular techniques: DNA isolation: Isolates were overnight subcultured in brain heart infusion broth and pelleted by centrifugation at 5,000 rpm for $15 \mathrm{~min}$, resuspended in $185 \mu \mathrm{l}$ of TE buffer (20 mMTris chloride, 2 mM EDTA [PH 8.0]) with 15 $\mu \mathrm{l}$ of recombinant lysostaphin $(15 \mathrm{mg} / \mathrm{ml}$; Sigma, St. Louis, Mo.) and incubated at $37^{\circ} \mathrm{C}$ for $30 \mathrm{~min}$, 
then boiled at $95^{\circ} \mathrm{C}$ for 15 minutes. After cooling to room temperature, the suspension was centrifuged at 10,000 rpm for 5 minutes, and the supernatant was used directly as template for PCR reactions[33].

Detection of nuc and mecA genes: Multiplex PCR was used for detection of the nuc (S. aureus species-specific) and mec $A$ (methicillin-resistance) genes[34].

Briefly, primers mecA-1 (5'-GGGATCATAGCG TCATTATTC-3') and mecA-2 (5'-AACGATTGTGAC ACGATAGCC-3') for the gene mecA and nuc-1 (5'-TCAGCAAATGCATCACAAACAG-3') and nuc-2 (5'-CGTAAATGCACTTGCTTCAGG-3') for the gene nuc were used in a multiplex $P C R$ reaction on a T100'TM Thermal Cycler (BioRad, USA) under the following conditions: an initial 5-min denaturation step at $95^{\circ} \mathrm{C}$, followed by 30 cycles of $1 \mathrm{~min}$ of denaturation at $95^{\circ} \mathrm{C}, 1 \mathrm{~min}$ of annealing at $59^{\circ} \mathrm{C}$, and 2 min of extension at $72^{\circ} \mathrm{C}$; with a final extension step at $72^{\circ} \mathrm{C}$ for $10 \mathrm{~min}$. PCR products were analyzed by electrophoresis on ethidiumbromidestained $2 \%$ agarose gels. The sizes of the amplicons were 530bp and 280bp for mecA and nuc genes, respectively. S. aureus ATCC 33592 was used as positive control for mecA and nuc genes and $S$. aureus ATCC 29213 was used as positive control for only nuc gene, while S. epidermidis DSM 20044 was used as negative control [34, 35].

Detection of the capsular polysaccharide locus type 5 and 8: For identification of the capsular polysaccharide locus type that define the CP5 and CP8 serotypes, a monoplex PCR was performed with primers specific for the variable segment of the cap locus (cap5: 5'-GAAAGTGAACGATTAGTAGAA-3',5'-GTACG AAGCGTTTTGATAGTT-3"; cap8: 5'-GTGGGATTT TTGTAGCTTTT- 3', 5'-CGCCTCGCTATATGAACTAT - 3") as described by Goerke et al., 2005 [36]. The sizes of the amplicons were 532bp and 437bp for cap5 and cap8 genes, respectively.

\section{Antimicrobial susceptibility testing}

S. aureus susceptibility to penicillin, ampicillin, oxacillin, erythromycin, clindamycin, gentamicin, cefoxitin, amikacin, doxycycline, ciprofloxacin, rifampin and trimethoprim/sulfamethoxazole were determined using the disk diffusion method in accordance with standards recommended by the CLSI [32]. All disks were purchased from HiMedia Laboratories Pvt. Limited, India, and S. aureus ATCC 33592 was used as a quality control strain. Multidrug resistance (MDR) phenotype was defined as resistance of $S$. aureus isolates to three or more district antimicrobial classes used in this study.

\section{Statistical analysis}

The results were tabulated, encoded and statistically analyzed using Statistical Package for Social Sciences (SPSS ${ }^{\circledR}$ ) program version 17 (Chicago, IL, USA). Fisher's exact 2-tailed and Pearson's $\chi^{2}$ tests were used for categorical variables to compare frequencies of $S$. aureus positivity and negativity and MRSA positivity and negativity. Likewise, percentages as of antibiotic resistance profiles of MRSA and MSSA were compared with Pearson's $\chi^{2}$ or Fisher's exact test as appropriate. A $P$ value of less than 0.05 was considered significant.

\section{Results}

In this study, 140 HCWs in three main hospitals in Gaza strip were screened for S. aureus and MRSA nasal colonization. The demographic characteristics of the participants are recorded in Table 1. Participants included 104 males and 36 females (ratio 1:2.9) and the majority (80\%) were from surgery and medicine departments. Also, the majority of them (92.8\%) have work experience ranged from 1-15 years.

Of the $140 \mathrm{HCW}$ tested in this study, 59 (42.1\%) were positive for S. aureus by phenotypic 
Table 1. Distribution of the study population by gender, hospital, ward and work experience.

\begin{tabular}{|c|c|c|}
\hline & & No. (\%) \\
\hline \multirow{3}{*}{ Gender } & Males & $104(74.3)$ \\
\hline & Females & $36(25.7)$ \\
\hline & Total & $140(100)$ \\
\hline \multirow{4}{*}{ Hospital } & European & $58(41.4)$ \\
\hline & Al-Shefa & $37(26.4)$ \\
\hline & Odwan & $45(32.1)$ \\
\hline & Total & $140(100)$ \\
\hline \multirow{4}{*}{ Ward } & Surgery & 60 (42.9) \\
\hline & Medicine & $52(37.1)$ \\
\hline & ICUs & $28(20.0)$ \\
\hline & Total & $140(100)$ \\
\hline \multirow{4}{*}{$\begin{array}{c}\text { Work } \\
\text { experience }\end{array}$} & $1-5$ years & $64(45.7)$ \\
\hline & 6-15 years & $66(47.1)$ \\
\hline & $>15$ years & $10(7.1)$ \\
\hline & Total & 140 (100) \\
\hline
\end{tabular}

characteristics and analysis of nuc gene (Table 2, Figure 1). Screening for MRSA by testing for oxacillin (phenotypically) and analysis of the mecA gene by PCR revealed that $32(54.2 \%)$ of the S. aureus isolates were MRSA and 27 (45.8\%) were MSSA. The overall colonization prevalence was $42.1 \%$ for $S$. aureus and $22.9 \%$ for MRSA (Table 2, Figure 1).

As stated in Table 2, the highest prevalence of 5 . aureus colonization occurred in medicine ward (23 of 59 [44.2\%]), followed by surgery ward (25 of $59[41.7 \%])$. However, in regard to the work experience, the highest prevalence of $S$. aureus colonization was happened in HCWs who had work experience more than 15 years. There were no statistically significant differences in the rates of $S$. aureus carriage between males and females $(P=0.395)$, or among European, Al Shifa and Odwan hospitals $(P=0.554,0.747$, and 0.775 respectively). However, a statistically significant

ICUs: Intensive Care Units.

Table 2. Distribution of S. aureus that isolated from HCWs according to gender, hospital, ward and work experience.

\begin{tabular}{|c|c|c|c|c|c|}
\hline & & & & & \\
\hline & & $\begin{array}{l}\text { Positive } \\
\text { No. (\%) }\end{array}$ & $\begin{array}{c}\text { Negative } \\
\text { No. (\%) }\end{array}$ & $\begin{array}{c}\text { Total } \\
\text { No. (\%) }\end{array}$ & P-value \\
\hline \multirow{3}{*}{ Gender } & Males & $46(44.2)$ & $58(55.8)$ & $104(100)$ & 0.395 \\
\hline & Females & $13(36.1)$ & $23(63.9)$ & $36(100)$ & \\
\hline & Total & $59(42.1)$ & 81 (57.9) & $140(100)$ & \\
\hline \multirow{4}{*}{ Hospital } & European & $24(41.4)$ & 34 (58.6) & $58(100)$ & 0.554 \\
\hline & Al-Shefa & $15(40.5)$ & $22(59.5)$ & $37(100)$ & 0.747 \\
\hline & Odwan & $20(44.5)$ & $25(55.6)$ & $45(100)$ & 0.775 \\
\hline & Total & $59(42.1)$ & 81 (57.9) & $140(100)$ & \\
\hline \multirow{5}{*}{ Ward } & Surgery & $25(41.7)$ & $35(58.3)$ & $60(100)$ & $0.001 *$ \\
\hline & Medicine & $23(44.2)$ & $29(55.8)$ & $52(100)$ & $0.011 *$ \\
\hline & ICUs & $11(39.3)$ & $17(60.7)$ & $28(100)$ & 0.925 \\
\hline & Total & $59(42.1)$ & 81 (57.9) & $140(100)$ & \\
\hline & 1-5 years & $18(28.1)$ & $46(71.9)$ & $64(100)$ & 0.644 \\
\hline \multirow{3}{*}{ Work experience } & $6-15$ years & $33(50)$ & $33(50)$ & $66(100)$ & 0.279 \\
\hline & $<15$ years & $8(80)$ & $2(20)$ & $10(100)$ & $0.002^{*}$ \\
\hline & Total & $59(42.1)$ & $81(57.9)$ & $140(100)$ & \\
\hline
\end{tabular}

* Statistical significant difference in prevalence of S. aureus among HCWs according to type of ward.

$¥$ Statistical significant difference in prevalence of $\mathrm{S}$. aureus among HCWs according to work experience. 


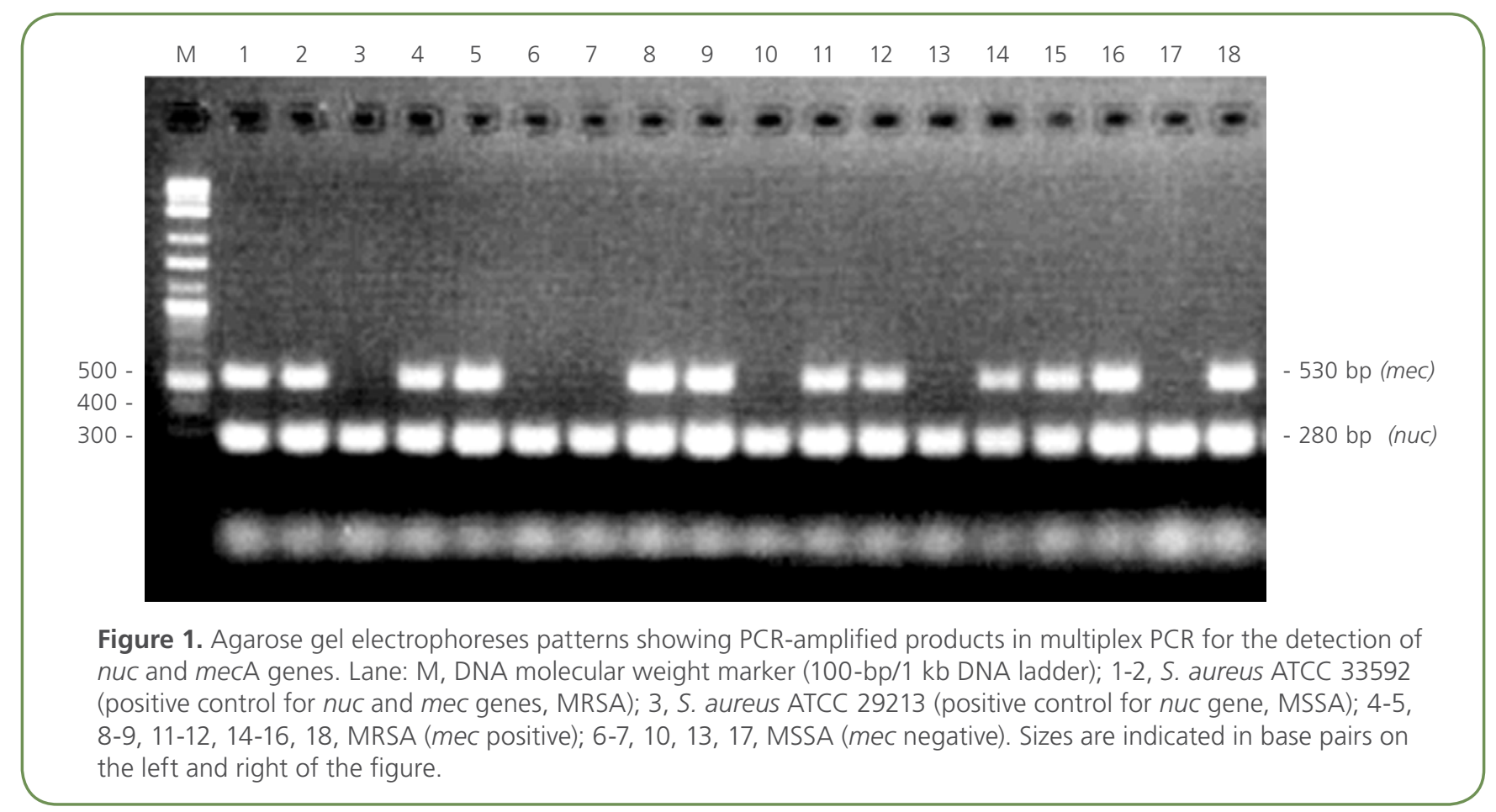

differences were found between departments, where surgery and medicine departments had higher rate than ICUs departments $(P=0.001$, $0.011)$, and between years of work experience of participants $(P=0.002)$.

As reported in Table 3 and according to the type of ward and hospital, the highest prevalence of MRSA colonization occurred in surgery department (20 of 25 [80\%]), followed by Al Shifa hospital (9 of 15 [60\%]). The prevalence of MRSA colonization was significantly higher in surgery department $(80 \%, P$ $=0.003)$, while there were no statistically significant differences in the rates of MRSA carriage according to hospital, gender or length of HCWs employment $(P=0.827,0.974$, and 0.58 respectively). Although the HCWs with work experience longer than 15 years had the highest prevalence of $S$. aureus carriage (80\%), they had the lowest prevalence of MRSA colonization (37.5\%). Finally, HCWs who worked in the surgery department were more likely to harbor MRSA than those who worked in other areas.
All S. aureus isolates tested were susceptible to vancomycin and doxycycline. The majority of $S$. aureus isolates were resistant to penicillin (96.6\%) and ampicillin (93.2\%). Rates of resistance to other antibiotics were $61 \%$ to erythromycin and cefoxitin, $59.3 \%$ to clindamycin, $30.5 \%$ to trimethoprim/ sulfamethoxazole (SXT), 27.1 to ciprofloxacin, $20.3 \%$ to gentamicin, $15.3 \%$ to rifampin, and $10.2 \%$ to amikacin [Figure 2]. Resistance to multiple classes of antimicrobials was found among both MRSA and MSSA, including $\beta$-lactams, macrolides, aminoglycosides, fluoroquinolones, and tetracyclines. Overall, MRSA strains exhibited complete resistance to penicillin, oxacillin and cefoxitin (100\%), and nearly complete resistance to ampicillin (96.9\%). Meanwhile, high resistance was also observed to erythromycin (75\%), clindamycin (75\%), SXT (56.2\%), ciprofloxacin (43.8\%), and moderately to gentamicin (37.5\%), rifampin (28.1\%) and amikacin (15.6\%). MSSA strains also exhibited nearly complete resistance to penicillin (92.6\%) and ampicillin (88.9\%). Resistance rate to erythromycin, 
Table 3. Distribution of MRSA and MSSA that isolated from HCWs according to gender, hospital, ward and work experience.

\begin{tabular}{|c|c|c|c|c|c|}
\hline Ward & & $\begin{array}{c}\text { MRSA } \\
\text { No. (\%) }\end{array}$ & $\begin{array}{c}\text { MSSA } \\
\text { No. (\%) }\end{array}$ & $\begin{array}{l}\text { Total } \\
\text { No. (\%) }\end{array}$ & $P$-value \\
\hline \multirow{3}{*}{ Gender } & Males & $25(54.3)$ & $21(45.7)$ & $46(100)$ & 0.974 \\
\hline & Females & $7(53.8)$ & $6(46.2)$ & $13(100)$ & \\
\hline & Total & $32(54.2)$ & $27(45.8)$ & $59(100)$ & \\
\hline \multirow{4}{*}{ Hospital } & European & $12(50)$ & $12(50)$ & $24(100)$ & 0.827 \\
\hline & Al-Shefa & $9(60)$ & $6(40)$ & $15(100)$ & \\
\hline & Odwan & $11(55)$ & $9(45)$ & $20(100)$ & \\
\hline & Total & $32(54.2)$ & $27(45.8)$ & $59(100)$ & \\
\hline \multirow{4}{*}{ Ward } & Surgery & $20(80)$ & $5(20)$ & $25(100)$ & $0.003^{*}$ \\
\hline & Medicine & $8(34.8)$ & $15(65.2)$ & $23(100)$ & \\
\hline & ICUs & $4(36.4)$ & $7(63.6)$ & $11(100)$ & \\
\hline & Total & $32(54.2)$ & $27(45.8)$ & $59(100)$ & \\
\hline \multirow{4}{*}{ Work experience } & 1-5 years & $11(61.1)$ & 7 (38.9) & $18(100)$ & 0.580 \\
\hline & $6-15$ years & $18(54.5)$ & $15(45.5)$ & $33(100)$ & \\
\hline & $>15$ years & $3(37.5)$ & $5(62.5)$ & $8(100)$ & \\
\hline & Total & $32(54.2)$ & $27(45.8)$ & $59(100)$ & \\
\hline
\end{tabular}

*Statistically significant difference in prevalence of MRSA and MSSA among HCWs according to type of ward

clindamycin, ciprofloxacin and amikacin was 44.4\%, $40.7 \%, 7.4 \%$, and $3.7 \%$ respectively. However, none of the MSSA strains $(0 \%)$ were resistant to oxacillin, gentamicin, SXT, and rifampin along with aforementioned vancomycin and doxycycline. The resistance rates of MRSA isolates to erythromycin, clindamycin, gentamicin, cefoxitin, ciprofloxacin,

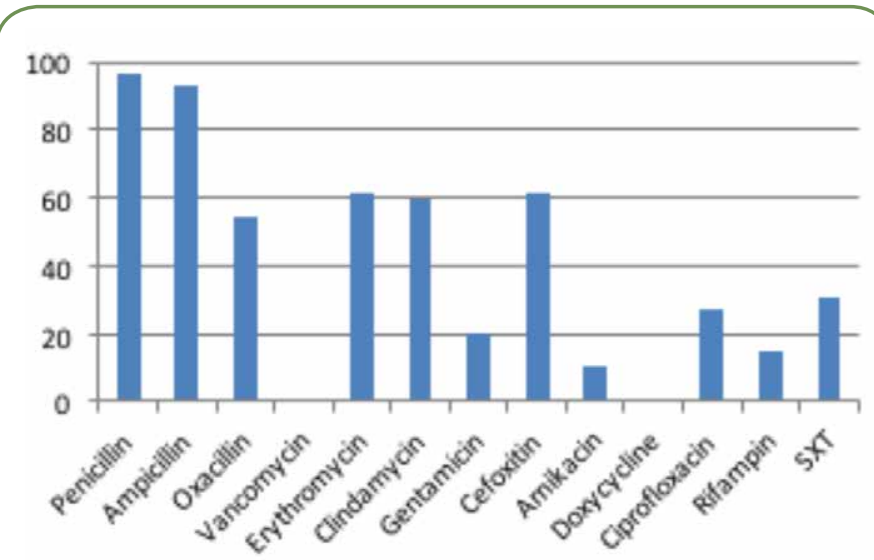

Figure 2. Antibiotic resistant pattern of $S$. aureus isolated from health care workers to the common used antibiotics in Gaza hospitals.SXT: Trimethoprim/sulfamethoxazole. rifampin, and SXT were significantly higher than those among MSSA isolates ( $P$ value $<0.05$ ) (Figure 3 ).

Multidrug resistance (MDR) among $S$. aureus isolates defined as resistance for $\beta$-lactam plus 2 or more antibiotics used in this study. Nearly $61 \%$

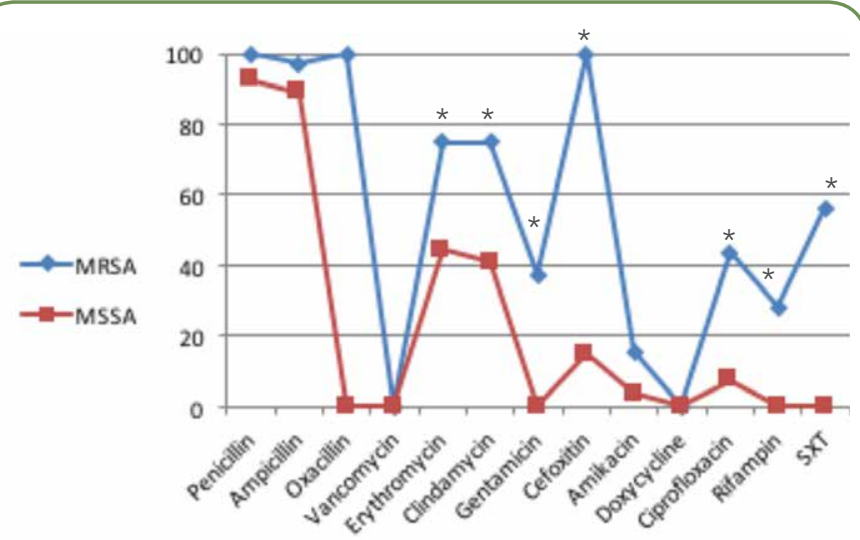

Figure 3. Antibiotic resistant pattern of MRSA and MSSA isolates from health care workers to the common used antibiotics in Gaza hospitals.

* Statistically significant difference in resistance pattern between MRSA and MSSA (P-value < 0.05).

MRSA: Methicillin resistant S. aureus; MSSA: Methicillin sensitive S. aureus; SXT: Trimethoprim/sulfamethoxazole. 
Table 4. Antibiogram resistance typing of the 32 MRSA isolates.

\begin{tabular}{|c|c|c|}
\hline Resistance pattern* & $\begin{array}{c}\text { Resistance } \\
\text { type }\end{array}$ & $\begin{array}{c}\text { No. of } \\
\text { isolates (\%) }\end{array}$ \\
\hline Amp & I & $5(15.6)$ \\
\hline Amp, Cip & II & $2(6.25)$ \\
Amp, R & III & $1(3.12)$ \\
\hline Amp, Ery, Cli, Cip & IV & $7(21.88)$ \\
\hline Amp, Ery, Cli, Cip, SXT & V & $7(21.88)$ \\
\hline Amp, Ery, Cli, Gent, R, & VI & $6(18.75)$ \\
\hline STX & & \\
\hline Amp, Ery, Cli, Gent, Ak, & VII & $4(12.5)$ \\
STX & & \\
\hline
\end{tabular}

*Amp, Ampicillin; Cip, Ciprofloxacin; R, Rifampin; Ery, Erythromycin; Cli, Clindamycin; SXT, Trimethoprim/ sulfamethoxazole; Gent, Gentamicin; Ak, Amikacin.

of isolates were noted to be MDR. Among MRSA isolates there were 24 out of 32 (75\%) MDR, while in MSSA there were 12 out of 27 (44.44\%) MDR. There was higher rate of MDR-MRSA in comparison to the MDR-MSSA. Seven different groups of antibiotypes were identified. Four of them were multiple resistant antibiotypes (resistant to 3 or more antimicrobial agents) and accounted for $75 \%$ of all S. aureus isolates (Table 4).

All $S$. aureus isolates were classified as containing either cap5 (33 isolates [55.9\%]) or cap8 (26 isolates [44.1\%]). There was higher rate of cap5 among MRSA and MSSA than cap8 $(28.8 \%$ and $27.1 \%$ vs. $25.4 \%$ and $18.6 \%$ respectively) (Table 5 ).

\section{Discussion}

The main route of transmission of $S$. aureus and MRSA in the hospital setting is considered to be from patient to patient via the contaminated hands of HCWs. They have been implicated as the source of infection in a number of published outbreak reports around the world [37]. Data regarding S. aureus and MRSA in Palestine are very scant. Previous reports
Table 5. Capsule polymorphism (capsular types 5 and 8) of MRSA and MSSA HCWs isolates

\begin{tabular}{|c|c|c|}
\hline Bacteria & cap5 (\%) & cap8 (\%) \\
\hline MRSA & $17(28.8)$ & $15(25.4)$ \\
\hline MSSA & $16(27.1)$ & $11(18.6)$ \\
\hline S. aureus & $33(55.9)$ & $26(44.1)$ \\
\hline
\end{tabular}

have mostly focused on Northern Palestine [28] and the West Bank [16], with absence or relatively little details regarding epidemiology of $S$. aureus in HCWs. Recently, a community-based nasal carriage among healthy children and hospital-based studies addressed the epidemiology of $S$. aureus and MRSA in Gaza Strip for the first time[29,30]. In this study, we described the nasal colonization rate of S. aureus and MRSA among a convenience sample of HCWs in three Gaza hospitals. To the best of our knowledge, this is the first report describing the prevalence of $S$. aureusc olonization and their resistance profiles among HCWs in the Gaza Strip.

The overall nasal carriage $S$. aureus prevalence in this study was $42.1 \%$. Of these, $54.2 \%$ were MRSA (22.9\% among the study population). Estimates of HCWs colonization rates from the worldwide literature vary widely depending on the country, hospital specialty and setting (endemic, non-endemic or outbreak) [37]. Our findings in the present study is comparable to the findings of other studies of $S$. aureus colonization rates among HCWs in developing countries as in Iran (31\%)[20]Libya (39\%) [22], Pakistan (48\%) [23], India (50\%) [24] or in developed countries as in Germany (33.8\%) [21] and USA (30\%, 43.8\%) [12,19]. However, the nasal carriage rate in this study was higher than those found in other studies that conducted in Arabic countries and other developing countries as seen in a recent publications from Libya (12.4\%) [38], India (17.5\%) [39], West Bank of Palestine (20.8\%) [16] and Kuwait (21\%) [14]. Yet, other recent studies from Saudi Arabia (76\%) [10], Taiwan (67.2\%) [26] 
and Senegal (56.1\%) [25] showed nasal colonization among HCWs higher than our findings. A plausible explanation for the differences in the colonization rates of $S$. aureus and MRSA between different countries and even some time in the same country may be due in part to differences in sample size or frequency of sampling and using different conventional and/or molecular methods among others.

In our study, there was no differences in the frequency of $S$. aureus between males and females HCWs or according to the type of hospital. Nevertheless, gender was found as a significant factor for $S$. aureus nasal colonization among HCWs in two studies in USA and Kenya where both studies indicated that males had higher rate of colonization [40]. On the other hand, there was significant higher rates of $S$. aureus colonization in surgery and medicine wards (Table 2 ) in comparison to ICUs departments.These results show those of Shibabaw et al., [18] who found that the highest rate of $S$. aureus carriers (35.7\%) and MRSA carriers (57.1\%) were among HCWs in pediatrics and surgery wards, respectively. A reason for this could be due to the higher exposure of HCWs in these departments to high numbers of patients and the crowd of these departments.

Our study revealed a high rate of MRSA nasal carriage among HCWs in this cohort (45.2\% out of S. aureus; $22.9 \%$ of all HCWs). To our knowledge, this is the highest rate of MRSA colonization reported in HCWs in Palestine. Kaibni et al., from West Bank, Palestine identified 13.9\% MRSA in 72 HCWs working in internal medicine department at Ramallah Governmental hospital [16]. All other reports of MRSA prevalence in Palestine have involved nasal carriage among healthy personnel who have not contact with hospital environment or hospital-based studies [16,28-30,41], although notably, $45 \%$ of the $S$. aureus isolates from the Gaza carriage study were methicillin-resistant [30]. Other studies from Arabic countries and worldwide showed lower prevalence of MRSA among HCWs as in Jordan (10.1\%) [13], in USA (12\%, 15.2\%) $[12,19]$, in Iran (17.2\%) [20] and in Libya (36.8\%) [22]. Interestingly, a higher prevalence (73\%) of MRSA than our findings was detected among HCWs in a recent study conducted in Saudi Arabia [10]. Therefore, the MRSA frequencies described in this report may be considered among the highest described in the region thus far. An important findings of our study is the highest occurrence of MRSA among HCWs in surgery departments (80\%, $P=0.003)$. This finding could be due to increased physical contact of surgeons and nurses with patients and over-crowding in surgery departments at our hospitals. Nasal carriage of S. aureus and MRSA in HCWs can reflect carriage of their 'own' isolate versus a transiently acquired isolate from patients. Accordingly, the MRSA prevalence rate detected here among HCWs could reflect a high MRSA rate in hospitalized patients, as was recently investigated in Gaza [29] but also a high MRSA carriage rate in the community as was shown in a recent communitybased nasal carriage study in Gaza [30]. Both studies showed a predominant lineage of $S$. aureus, with spa type t223, ST22-MRSA-IVa. Likewise, molecular typing of $S$. aureus isolated from HCWs in Portugal revealed that $88.6 \%$ of these isolates belong to the same clone (ST22-MRSA-IV) [42]. We think that same clone is present among our HCWs isolates, however, this needs to be approved by molecular epidemiology typing methods such as PFGE, spa, SCCmec and MLST.

In our present study, the antibiogram pattern of all $S$. aureus isolates showed resistance to most of the antibiotics tested, mainly penicillin, ampicillin, erythromycin and clindamycin. Almost all MRSA and MSSA were resistant to penicillin and ampicillin. This pattern of resistance was shown in previous studies from Gaza Strip and West Bank $[16,28,29]$ and also mirrored others elsewhere[5,13,18,20,22-24]. High resistance to tested antibiotics could be mainly due to the excessive use, misuse, and great prescription 
of these medication in Gaza for both hospital and community acquired infections and also in agriculture and animal feeding. Moreover, the lack of an antibiotic policy and the availability of antibiotics sold without a medical doctor prescription in Palestine worsen the case. In view of the high resistance rates of $S$. aureus to these aforementioned antibiotics, treatment of $S$. aureus infections at our hospitals with these antibiotics may not be effective. On the other hand, all $S$. aureus isolates including both MRSA and MSSA, were susceptible to vancomycin and doxycycline, and mostly to amikacin and rifampin (89.8\%, $84.7 \%$ respectively). This could be due to the limited prescription and use of these antibiotics in Gaza Strip. So, the full sensitivity to vancomycin and doxycycline can work perfectly against $S$. aureus infections in our community and hospitals as indicated in recent studies in Gaza $[29,30]$.

The trimethoprim-sulfamethoxazole showed moderate activity against $S$. aureus isolates in general (69.5\%), but surprisingly, with $100 \%$ and $43.8 \%$ activity against MSSA and MRSA respectively (Fig. 2, 3). This picture contradict several studies reporting low rates of resistance to SXT against S. aureus isolated from HCWs with percentage of $1.9 \%, 4.7 \%$ and $21.4 \%$ [43], but in accordance with a hospital-based study in Gaza [29] and other study in China that revealed $93 \%$ resistance to SXT of HCWs nasal S. aureus isolates [43]. The MRSA isolates recovered in this study were resistant to quit high number of tested antimicrobial agents, and were found to be multidrug resistant (75\%) (Fig. 3, Table 4). They were significantly have higher resistance to erythromycin, clindamycin, gentamycin, amikacin, ciprofloxacin, rifampin and SXT than MSSA (Fig. 3). This finding contradict the results of a recent study from Jordan that showed MRSA isolates were susceptible to a broad range of tested antibiotics, and none were MDR [13]. Meanwhile, Kaibni et al., showed also less resistant to non- $\beta$ lactam antimicrobial agents but with $14.7 \%$ being MDR [16]. This percentage of MDR S. aureus nasal isolates is lower than our findings (75\%), yet, a study from Serbia showed $83.9 \%$ MDR S. aureus nasal carriage isolates from HCWs [5].

On regard to the capsular polymorphism, Table 5 showed that all $S$. aureus isolates in this study encoded cap5 or cap8 gene. Many previous studies reported the majority of human $S$. aureus strains (70-80\%) possess either cap5 and/or cap8 [44]. Nevertheless, a study from USA revealed that all pediatric isolates contained either cap5 or cap8 genes [45], while a recent study from Australia shows absence of cap8 and cap5 (non-typable strains) in $19.4 \%$ of their isolates [44]. Capsular polysaccharide plays an important role in the pathogenicity and immunogenicity of $S$. aureus, notably as antiphagocytic factor but also as a vaccine target. However, loss of capsule expression, may lead to $S$. aureus persistence in a chronically infected host as shown in chronic osteomyelitis patients [46].

Our study has several limitations. First, we may have over or under estimated the prevalence of $S$. aureus and MRSA nasal carriage in HCWs because this was a simple point prevalence study, using a convenience sample of subjects. Also, only nasal swabs were collected and assessed, and other body sites such as throat, axilla and rectum that could be colonized were not collected. Second, we did not use an enrichment step and just directly inoculated the swabs on culture media. So, this may underestimate the prevalence of $S$. aureus and MRSA among the study subjects. Likewise, molecular typing was not performed in this study, such that the clonality of the MRSA and MSSA strains cannot be assessed. Lastly, we were not able to determine whether the S. aureus and MRSA colonization state is transient, 
short-term, or persistent because we only screened the participants once and only one swab was collected.

Nevertheless, several themes of potential concern are raised by our findings. Most noteworthy is the high prevalence of MRSA, particularly among surgery departments. Also, the high resistance profile of MRSA against most common used antibiotics in our hospitals, and the high percentage of MDRMRSA strains. Further studies should address the molecular characterization and virulence factors of S. aureus isolated from HCWs in Gaza.

In conclusion, this study demonstrates high rate of MDR isolates of MRSA. These results highlight the critical need for screening and tracking MRSA among HCWs as well as patients and to monitor and manage the usage of antibiotics in the hospitals and community.

\section{Acknowledgments}

The author wants to thank all the healthcare workers of Al-Shifa, European and Odwan hospitals who participated in this study. Also, he would like to thank the German Academic Exchange Service (DAAD) short visit program, Germany and the Erasmus Mundus External Cooperation Window (EM ECW lot III), Belgium, EU for support of his work during his visits to the University Clinic of Muenster, Germany and the Free University of Brussels, Belgium.

\section{Conflict of interest}

The author declares that there is no conflict of interests to declare.

\section{References}

1. Song $Y$, Du X, Li T, Zhu Y, Li M. Phenotypic and molecular characterization of Staphylococcus aureus recovered from different clinical specimens of inpatients at a teaching hospital in Shanghai between 2005 and $2010 \mathrm{~J}$ Med Microbiol 2013;62(Pt 2):274-82.

2. Tokajian ST, Khalil PA, Jabbour D, Rizk M, Farah MJ, Hashwa FA, et al. Molecular characterization of Staphylococcus aureus in Lebanon. Epidemiol Infect 2010;138(5):707-12.

3. Nadig S, RamachandraRaju S, Arakere G. Epidemic meticillinresistant Staphylococcus aureus (EMRSA-15) variants detected in healthy and diseased individuals in India. J Med Microbiol 2010;59(Pt 7):815-21.

4. Du J, Chen C, Ding B, Tu J, Qin Z, Parsons C, et al. Molecular characterization and antimicrobial susceptibility of nasal Staphylococcus aureus isolates from a Chinese medical college campus. PLoS One 2011;6(11):e27328.

5. Cirkovic I, Stepanovic S, Skov R, Trajkovic J, Grgurevic A, Larsen AR. Carriage and Genetic Diversity of Methicillin-Resistant Staphylococcus aureus among Patients and Healthcare Workers in a Serbian University Hospital. PLoS One 2015;10(5):e0127347.

6. Tokajian, S. New epidemiology of Staphylococcus aureus infections in the Middle East. ClinMicrobiol Infect 2014;20(7):624-8.

7. Mediavilla JR, Chen L, Mathema B, Kreiswirth BN. Global epidemiology of community-associated methicillin resistant Staphylococcus aureus (CA-MRSA). CurrOpinMicrobiol 2012;15(5):588-95.

8. DeLeo, FR, Otto M, Kreiswirth BN, Chambers HF. Communityassociated meticillin-resistant Staphylococcus aureus. Lancet 2010;375(9725):1557-68

9. Albrich WC and Harbarth S. Health-care workers: source, vector, or victim of MRSA?. Lancet Infect Dis 2008;8(5):289-301.

10. Iyer A, Kumosani T, Azhar E, Barbour E, Harakeh S. High incidence rate of methicillin-resistant Staphylococcus aureus (MRSA) among healthcare workers in Saudi Arabia. J Infect Dev Ctries 2014;8(3):372-8.

11. von Eiff C, Becker K, Machka K, Stammer H, Peters G. Nasal carriage as a source of Staphylococcus aureus bacteremia. Study Group. N Engl J Med 2001;344(1):11-6.

12. Elie-Turenne MC, Fernandes $H$, Mediavilla JR, Rosenthal $M$, Mathema B, Singh A, et al. Prevalence and characteristics of Staphylococcus aureus colonization among healthcare professionals in an urban teaching hospital. Infect Control Hosp Epidemiol 2010;31(6):574-80.

13. Aqel AA, Alzoubi HM, Vickers A, Pichon B, Kearns AM. Molecular epidemiology of nasal isolates of methicillin-resistant Staphylococcus aureus from Jordan. J Infect Public Health 2015;8(1):90-7.

14. Dimitrov T, Udo EE, Grover S. Point surveillance of Staphylococcus 
aureus carriage among medical staff in Infectious Diseases Hospital, Kuwait. Med PrincPract 2003;12(3):139-44.

15. Rongpharpi SR, Hazarika NK, Kalita H. The prevalence of nasal carriage of Staphylococcus aureus among healthcare workers at a tertiary care hospital in Assam with special reference to MRSA. J Clin Diagn Res 2013;7(2):257-60.

16. Kaibni MH, Farraj MA, Adwan K, Essawi TA.Communityacquired meticillin-resistant Staphylococcus aureus in Palestine. J Med Microbiol 2009;58(Pt 5):644-7.

17. Ahmed MO, Elramalli AK, Amri SG, Abuzweda AR, Abouzeed YM. Isolation and screening of methicillin-resistant Staphylococcus aureus from health care workers in Libyan hospitals. East Mediterr Health J 2012;18(1):37-42.

18. Shibabaw A, Abebe T, Mihret A. Nasal carriage rate of methicillin resistant Staphylococcus aureus among Dessie Referral Hospital Health Care Workers; Dessie, Northeast Ethiopia.Antimicrob Resist Infect Control 2013;2(1):25.

19. Ibarra M, Flatt T, Van Maele D, Ahmed A, Fergie J, Purcell K. Prevalence of methicillin-resistant Staphylococcus aureus nasal carriage in healthcare workers.Pediatr Infect Dis J 2008;27(12):1109-11.

20. Askarian M, Zeinalzadeh A, Japoni A, Alborzi A, Memish ZA. Prevalence of nasal carriage of methicillin-resistant Staphylococcus aureus and its antibiotic susceptibility pattern in healthcare workers at Namazi Hospital, Shiraz, Iran.Int J Infect Dis 2009;13(5):e241-7.

21. Kampf G, Adena S, Rueden H, Weist K. Inducibility and potential role of MecA-gene-positive oxacillin-susceptible Staphylococcus aureus from colonized healthcare workers as a source for nosocomial infections. J Hosp Infect 2003;54(2):124-9.

22. Zorgani A, Elahmer O, Franka E, Grera A, Abudher A, Ghengheah KS. Detection of meticillin-resistant Staphylococcus aureus among healthcare workers in Libyan hospitals. J Hosp Infect 2009;73:91-2.

23. Farzana K, Rashid Z, Akhtar N, Sattar A, Khan JA, Nasir B. Nasal carriage of staphylococci in health care workers: antimicrobial susceptibility profile. Pak J Pharm Sci 2008;21(3):290-4.

24. Goyal R, Das S, Mathur M.Colonisation of methicillin resistant Staphylococcus aureus among health care workers in a tertiary care hospital of Delh. Indian J Med Sci 2002;56(7):321-4

25. Fall C, Richard V, Dufougeray A, Biron A, Seck A, Laurent F, et al. Staphylococcus aureus nasal and pharyngeal carriage in Senegal.Clin Microbiol Infect 2014;20(4):O239-41.

26. Lin $Y C$, Lauderdale TL, Lin HM, Chen PC, Cheng MF, Hsieh KS, et al. An outbreak of methicillin-resistant Staphylococcus aureus infection in patients of a pediatric intensive care unit and high carriage rate among health care workers. J Microbiol Immunol Infect 2007;40(4):325-34.

27. Rongpharpi SR, Hazarika NK, Kalita $H$. The prevalence of nasal carriage of Staphylococcus aureus among healthcare workers at a tertiary care hospital in assam with special reference to MRSA. J ClinDiagn Res 2013;7(2):257-60.

28. Adwan K, Jarrar N, Abu-Hijleh A, Adwan G, Awwad E, Salameh Y. Molecular analysis and susceptibility patterns of methicillin- resistant Staphylococcus aureus strains causing communityand health care-associated infections in the northern region of Palestine. Am J Infect Control 2013;41(3):195-8.

29. Al Laham N, Mediavilla JR, Chen L, Abdelateef N, Elamreen FA, Ginocchio CC, et al. MRSA clonal complex 22 strains harboring toxic shock syndrome toxin (TSST-1) are endemic in the primary hospital in Gaza, Palestine.PLoS One 2015;10(3):e0120008.

30. Biber A, Abuelaish I, Rahav G, Raz M, Cohen L, Valinsky L, et al. A typical hospital-acquired methicillin-resistant Staphylococcus aureus clone is widespread in the community in the Gaza strip. PLoS One 2012;7(8):e42864.

31. Scarnato F, Mallaret MR, Croize' J. Incidence and prevalence of methicillin-resistant Staphylococcus aureus nasal carriage among healthcare workers in geriatric departments: relevance to preventive measures. Infect Control HospEpidemiol 2003;24:456-8.

32. Clinical and Laboratory Standards Institute. Approved standard: M02-A10. Performance standards for antimicrobial disk susceptibility tests; 10th ed. Clinical and Laboratory Standards Institute. 2009. Wayne, Pa.

33. Mathem B, Mediavilla J, and Kreiswirth BN. Sequence analysis of the variable number tandem repeat in Staphylococcus aureus protein A gene: spa typing. Methods MolBiol 2008;431:285305.

34. Becker K, Roth R, and Peters G. Rapid and specific detection of toxigenic Staphylococcus aureus: use of two multiplex PCR enzyme immunoassays for amplification and hybridization of staphylococcal enterotoxin genes, exfoliative toxin genes, and toxic shock syndrome toxin 1 gene. J Clin Microbiol 1998;36(9):2548-53.

35. Becker K, Pagnier I, Schuhen B, Wenzelburger F, Friedrich AW, Kipp $F$, et al. Does nasal cocolonization by methicillinresistant coagulase-negative staphylococci and methicillinsusceptible Staphylococcus aureus strains occur frequently enough to represent a risk of false-positive methicillin-resistant S. aureus determinations by molecular methods? J ClinMicrobiol 2006;44(1):229-31.

36. Goerke C, Esser S, Kuemmel M, Wolz C. Staphylococcus aureus strain designation by agrand cap polymorphism typing and delineation of agrdiversification by sequence analysis. Int J Med Microbiol 2005;295(2):67-75.

37. Hawkins G, Stewart S, Blatchford O, Reilly J. Should healthcare workers be screened routinely for meticillin-resistant Staphylococcus aureus? A review of the evidence. J Hosp Infect 2011;77(4):285-9.

38. Al-haddad OH, Zorgani A, Ghenghesh KS. Nasal carriage of multi-drug resistant Panton-Valentine leucocidin-positive methicillin-resistant Staphylococcus aureus in children in TripoliLibya. Am J Trop Med Hyg 2014;90(4):724-7.

39. M R, D'Souza M, Kotigadde S, Saralaya KV, Kotian MS. Prevalence of Methicillin Resistant Staphylococcus aureus Carriage amongst Health Care Workers of Critical Care Units in Kasturba Medical College Hospital, Mangalore, India. J ClinDiagn Res 2013;7(12):2697-700. 
40. Omuse G, Kariuki S, Revathi G. Unexpected absence of meticillin-resistant Staphylococcus aureus nasal carriage by healthcare workers in a tertiary hospital in Kenya. J Hosp Infect 2012;80(1):71-3

41. Al Laham NA. Prevalence of bacterial contamination in general operating theaters in selected hospitals in the Gaza Strip, Palestine. J Infect Public Health 2012;5(1):43-51.

42. Amorim ML, Vasconcelos C, Oliveira DC, Azevedo A, Calado E, Faria NA, et al. Epidemiology of methicillin-resistant Staphylococcus aureus (MRSA) nasal colonization among patients and healthcare workers in a Portuguese hospital: a preintervention study toward the control of MRSA.Microb Drug Resist 2009;15(1):19-26.

43. Du J, Chen C, Ding B, Tu J, Qin Z, Parsons C, et al. Molecular Characterization and Antimicrobial Susceptibility of Nasal Staphylococcus aureus Isolates from a Chinese Medical College Campus. PLoS ONE 2011;6(11):e27328.

44. Waryah CB, Gogoi-Tiwari J, Wells K, Costantino P, Al-Salami $H$, Sunagar $R$, et al. Serological versus molecular typing of surface-associated immune evading polysaccharide antigensbased phenotypes of Staphylococcus aureus. J Med Microbiol 2014;63(Pt 11):1427-31.

45. Sutter DE, Summers AM, Keys CE, Taylor KL, Frasch CE, Braun LE, et al. Capsular serotype of Staphylococcus aureus in the era of community-acquired MRSA. FEMS Immunol Med Microbiol 2011;63(1):16-24.

46. Lattar SM, Tuchscherr LP, Caccuri RL, Centrón D, Becker K, Alonso CA, et al. Capsule expression and genotypic differences among Staphylococcus aureus isolates from patients with chronic or acute osteomyelitis. Infect Immun 2009;77(5):196875 .

\section{Comment on this article:}
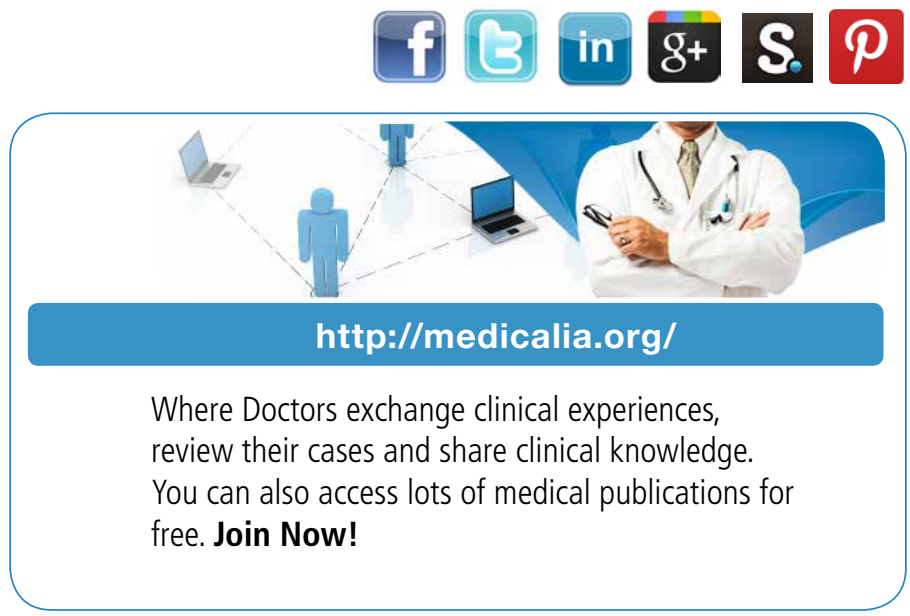

\section{Publish with iMedPub}

http://www.imed.pub

The Journal is an open access peer-reviewed journal that publishes scientific papers about all aspects of antimicrobials. The journal will publish original research articles, reviews, brief reports and case reports dealing with basic and clinical antibacterial agents, antiviral, antiprotozoals, antituberculuous, antifungal and antihelminthes agents.

All manuscripts must be prepared in English, and are subject to a rigorous and fair peer-review process. Accepted papers will immediately appear online.

The journal aims to advance the knowledge, attitude and the research of chemotherapy in the Arabic world in cooperation with international, national scientific and public societies as well as research centers with similar aims and objectives. 